\title{
Preparation of soluble conducting polymers
}

\author{
from Paul Calvert
}

CONDUCTING polymers have been the focus of a great deal of research in the last few years. The interest started with the discovery that acetylene could be polymerized to a shiny black film, polyacetylene (I in the figure), which became highly conducting when 'doped' by reaction with large amounts of strong oxidizing or reducing agent ${ }^{1-3}$. The conductivity is attributable to the ease of electron transport along the chain. Many other polymers with similarly conjugated double bonds have been found to conduct when doped.

The promise offered by these materials is of a class of conductors or semiconductors that would carry the benefits associated with polymers: cheapness, toughness and easy processing to fibres, films and coatings on a large scale. Unfortunately most of these convenient properties are lost in the conjugated systems whose chain rigidity and strong interchain forces make them insoluble infusible black powders. Recent months have seen the publication of a number of routes to solubilizing conducting polymers (see the figure).

One route is to prepare a flexible soluble precursor polymer which can be readily cast into films before conversion to polyacetylene. In principle, polyvinylchloride (PVC; II in the figure) should do this because it is degraded on heating by loss of $\mathrm{HCl}$ to leave a black polyacetylene-like material. However the result is not conducting when doped, apparently because removal of adjoining $\mathrm{H}, \mathrm{Cl}$ pairs at random will leave many isolated $\mathrm{Cl}$ or $\mathrm{H}$ atoms which break up the conjugated sequences. A way of avoiding this is to pair up the leaving groups beforehand. Jim Feast and his co-workers have produced a number of precursor polymers which lose cyclic compounds on heating and transform to polyacetylene ${ }^{4,5}$. The unnameable precursor polymer (III) is soluble in acetone and forms transparent films which, at $50-80^{\circ} \mathrm{C}$, lose hexafluoro- $O$-xylene and 70 per cent of their weight to leave black shiny dopable amorphous polyacetylene. Interest is centring on the fact that this material is different in structure and properties from polymer prepared by direct polymerization, but characterization of black insoluble films is no easy task.

Another approach to solubility is to modify the polymer to build enough flexibility into the chains to allow them to dissolve without losing conductivity. The results of copolymerization with small amounts of polymethylacetylene have been disappointing because conductivity rapidly decreased as side groups were added to the chain.

Workers at Bell Laboratories now report graft copolymers with acetylene chains of
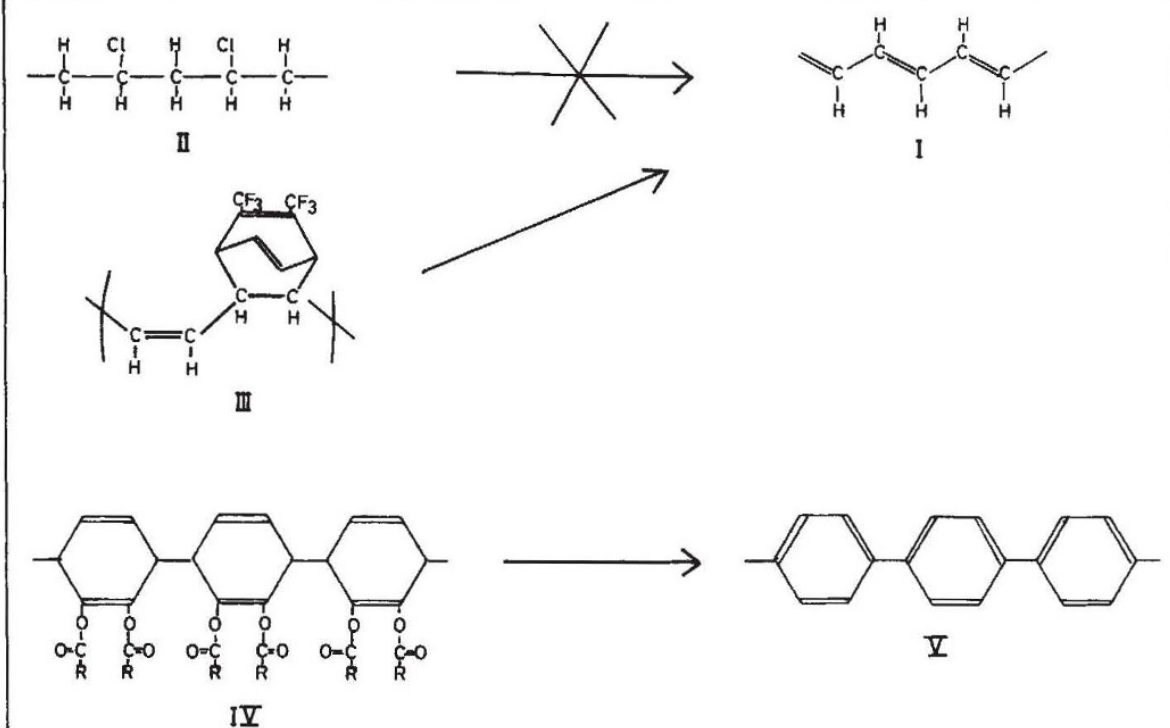

I

Various routes recently used in attempts to solubilize conducting polymers. I, polyacetylene; II, polyvinylchloride; III, precursor polymer; IV, esterified polycyclohexene; V, polyphenylene.

20,000 molecular weight attached to polyisoprene $^{6}$. These soluble polymers have a separate acetylene phase in the solid state and yield interesting spectroscopic data but there is no mention of conductivity. Galvin and Wnek $^{7}$ have described previously a similar system where acetylene is polymerized inside polyethylene to produce a conducting composite.

Polyacetylene has the disadvantage that it is not very stable to oxidative degradation. From this point of view polyphenylene is a much more attractive polymer but it has only been available so far as an intractable powder of very short chain length. Ballard and co-workers at ICI Runcorn are now reporting ${ }^{8}$ a route through a soluble precursor polymer, an esterified polycyclohexene (IV) which converts to polyphenylene (V) on heating to $240^{\circ} \mathrm{C}$.

This will be of great interest because the polyphenylenes studied to date have been poorly characterized materials with short chain lengths and it offers the exciting prospect of being able to control the chain length and composition and study the effect on properties. An added bonus is that there is a bacterial fermentation route from benzene to the precursor. This may not materially affect the final polymer but it has enabled Ballard and colleagues to perform the remarkable feat of joining both the biotechnology and conducting polymer bandwagons at once.

Recently published studies on another of these polymers, polyphenylenesulphide, at Allied Chemical, illustrates the complexity of solubilizing them ${ }^{9}$. Strange effects were found when this polymer was doped with arsenic pentafluoride contaminated with arsenic trifluoride. $\mathrm{AsF}_{3}$ had little effect on the polymer itself but seemed to be a strong plasticizing agent for the $\mathrm{AsF}_{5}$-doped state. Using liquid $\mathrm{AsF}_{3}$ Frommer and his colleagues now report that the doped polymer dissolves to a blue solution from which they can cast tough conducting films. When cast the film will not redissolve because of cross-linking. It seems often to be the case that such powerful dopants also cause side reactions and cross-linking.

This year a mood of realism has been spreading over this rapidly growing field. Various applications for conducting polymers have been proposed in the past, including solar cells, semiconductor devices and batteries, but these are still a long way off and a polymeric replacement for copper wires looks unlikely. Those interested in silicon semiconductors have been pointing out that the polymers are impure unstable uncharacterized junk compared with single-crystal silicon. The availability of soluble materials that can be purified and characterized should improve this state of affairs.

Paul Calvert is at the School of Chemistry and Molecular Sciences, University of Sussex, Falmer, Brighton BNI 9QJ.

\footnotetext{
1. See Calvert, P. Nature 284, 213 (1980).

2. Wegner, G. Agnew. Chem. int. Edn 20, 361 (1981)

3. Baughman, R.H. et al. Chem. Rev. 82, 209 (1982)

4. Edwards, J.H. \& Feasi, W.J. Polymer 21, 595 (1980).

5. Feast, J. Polymer (submitted).

6. Bates, F.S. \& Baker, G.L. Macromolecules 16, 704 (1983)

6. Bates, F.S. \& Baker, G.L. Macromolecules 16, $704(1983)$
7. Galvin, M.E. \& Wnek, G.E. Polymer 23, 795 (1982).

8. Ballard, D.G.H., Courtis, A., Shirley, I.M. \& Taylor, S.C. Chem. Commun. (submilted).

9. Frommer, J.E., Elsenbaumer, R.L.\& Chance, R.R. ACS ORPL Preprints 48, 552 (1983).
} 